BI-TP 2010/09

TIFR/TH/10-08

\title{
The QCD Phase Structure at High Baryon Density
}

\author{
P. Castorina ${ }^{a}$, R. V. Gavai ${ }^{b, c}$ and H. Satz ${ }^{c}$ \\ ${ }^{a}$ Dipartimento di Fisica, Università di Catania, and INFN Sezione di Catania, Italy \\ ${ }^{b}$ Tata Institute for Fundamental Research, Mumbai, India \\ ${ }^{c}$ Fakultät für Physik, Universität Bielefeld, Germany
}

\begin{abstract}
:
We consider the possibility that color deconfinement and chiral symmetry restoration do not coincide in dense baryonic matter at low temperature. As a consequence, a state of massive "constituent" quarks would exist as an intermediate phase between confined nuclear matter and the plasma of deconfined massless quarks and gluons. We discuss the properties of this state and its relation to the recently proposed quarkyonic matter.
\end{abstract}

\section{Introduction}

Dense nuclear matter has been a subject of great interest for a long time and from quite different points of view. Starting with the medium which makes up heavy nuclei, an increase of density led to the regime of neutron stars, and once the quark infrastructure of nucleons was introduced, to possible quark cores of such stellar states [1,2]. Much subsequent work has then addressed the formation of color superconducting quark matter [1,3]. In the present paper, we want to return to the idea that besides these states, at high baryon density a plasma of massive deconfined quarks can still exist as yet another form of strongly interacting matter [4-11].

The essential features of hadrons are color confinement and spontaneous chiral symmetry breaking. The former binds colored quarks interacting through colored gluons to colorneutral hadrons. The latter brings in pions as Goldstone bosons and gives the essentially massless quarks in the QCD Lagrangian a dynamically generated effective mass. Both features will come to an end in hadronic matter at sufficiently high temperatures and/or baryon densities, though not a priori simultaneously. However, rather basic arguments suggest that chiral symmetry restoration occurs either together with or after color deconfinement [12].

It thus appears conceivable that QCD could lead to a three-state phase structure as function of the temperature $T$ and the baryochemical potential $\mu$, as shown in Fig. 1] [6]. In such a scenario, color deconfinement would result in a plasma of massive "dressed" quarks; the only role of gluons in this state would be to dynamically generate the effective 
quark mass, maintaining spontaneous chiral symmetry breaking. At still higher $T$ and/or $\mu$, this gluonic dressing of the quarks would then "evaporate" or "melt", leading to a plasma of deconfined massless quarks and gluons: the conventional QGP, with restored chiral symmetry. Evidently, this view of things ignores the possibility of bosonic diquark binding and condensation as well as that of the color superconductivity states which could result as a consequence; we shall return to these aspects later on.

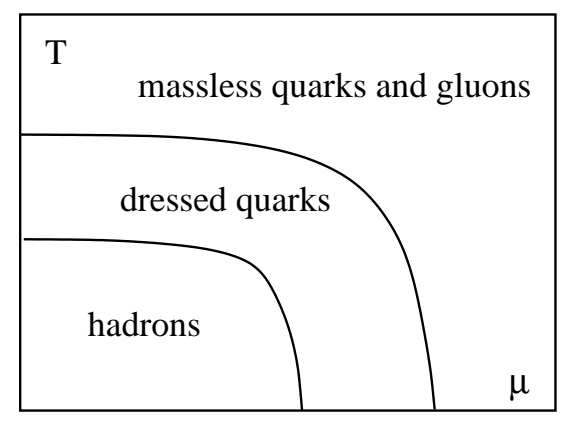

Figure 1: Three-state scenario of QCD matter [6]

As already noted, the possible existence of a phase of quarks with a dynamically generated effective mass has often been considered before [4-11]. However, in recent years finite temperature lattice QCD investigations have provided an impressive amount of further information which can be used in determining the properties of constituent quarks [13, 14,16. In addition, the proposal for a state of "quarkyonic" matter in the limit of a large number of color degrees of freedom [17, 18] has also renewed the interest in a possible constituent quark phase.

The basic idea in our considerations will be that a medium of constituents endowed with an intrinsic spatial scale $L$ can only exist as long as its density remains below $1 / L^{3}$. In strong interaction physics, this had first led to the prediction of an upper density limit for mesonic matter [19]. In the past decades it has found a quantitative formal basis in percolation theory [20]. The natural starting point is thus the determination of the intrinsic scales for the given medium.

Hadronic interactions lead to two intrinsic scales, one on the hadronic and the other on the quark level. Both can be expressed in different ways, and we shall elaborate on this. However, since we want to resort to geometric arguments, size parameters are the most useful. One scale then is given by the confinement radius $R_{h} \simeq 1 \mathrm{fm}$, defining the range of the strong force and thus also the size of hadrons. As long as the density $n$ of the medium remains below $n_{c}^{h} \simeq 1 / V_{h}^{3}$, with $V_{h}=4 \pi R_{h}^{3} / 3$, it is expected to be of hadronic nature. Inside a hadron, the valence quark constituents acquire a dynamically generated effective mass $M_{q}$ and size $R_{q}$. We shall show in the next section that both theoretical and experimental studies indicate $M_{q} \simeq 0.3-0.4 \mathrm{GeV}$ and $R_{q} \simeq 0.3 \mathrm{fm}$. In the density region $n_{c}^{h}<n<n_{c}^{q} \simeq 1 / V_{q}^{3}$, with $V_{q}=4 \pi R_{q}^{3} / 3$, we then expect a plasma of deconfined massive quarks, while for $n>n_{c}^{q}$, the medium will become the conventional quark-gluon plasma, with pointlike massless quarks and gluons as constituents. If both the parameters $R_{h}$ and $R_{q}$ were independent of temperature and baryon density, the critical points in the 
phase diagram of Fig. 1 could thus be easily defined in terms of corresponding percolation points.

However, it is well-known that the latter premise is not generally correct. Lattice studies at or near $\mu=0$ have shown that here color deconfinement and chiral symmetry restoration coincide [21. Hence in a medium of vanishing baryon density, the mass of the constituent quark vanishes at the deconfinement point $T_{c}$. This is in accord with the formation of the quark dressing through a polarization cloud in the surrounding gluonic medium; as the temperature approaches $T_{c}$, the screening radius defining cloud size decreases rapidly, and at $T_{c}$, the cloud has "evaporated", leaving pointlike quarks and gluons. There thus remains only one scale, which defines the simultaneous onset of color deconfinement and chiral symmetry restoration. Since the physical reason for this is apparently the presence of a hot gluonic environment, there is little reason to expect a similar behavior at low $T$ and large $\mu$. The aim of our study is therefore to introduce in this region an intermediate plasma of massive quarks, separating hadronic matter and QGP by a state of quark deconfinement and broken chiral symmetry.

We begin by considering nature and properties of constituent quarks, followed by a discussion of deconfinement and chiral symmetry restoration as consequence of the density of the relevant constituents. With these results as input, we then study the possible structure of a massive quark phase, and finally compare it to that of quarkyonic matter.

\section{Constituent Quarks}

There are two different regimes for the quark infrastructure of hadrons, depending how we probe. Relatively hard probes, such as deep inelastic lepton-hadron scattering or hadronhadron interactions at large momentum transfer, lead to massless pointlike quarks and gluons. In this regime, the parton model with hadronic quark and gluon distribution functions provides a suitable description. On the other hand, soft interactions, as seen in minimum bias proton-proton or pion-proton interactions, suggest that mesons/nucleons consist of two/three "constituent" quarks having a a size of about $0.3 \mathrm{fm}$ and a mass of about $0.3-0.4 \mathrm{GeV}$. Here many features are well accounted for by the additive quark model [22]. We can thus imagine that inside a hadron, a quark polarizes the gluon medium in which it is held through color confinement, and the resulting gluon cloud forms the constituent quark mass $M_{q}$ [23, 24].

This picture is today found to be quite compatible with heavy quark correlation studies in finite temperature lattice QCD at vanishing baryon density. By evaluating Polyakov loop correlations in a QCD medium of two or three light quark flavors below deconfinement $\left(T<T_{c}\right)$, one obtains the free energy $F(r, T)$ as function of the quark separation distance $r$. In the low temperature limit, $F(r, T=0)$ saturates beyond a separation of $r \simeq 1.5 \mathrm{fm}$, converging to the value $F(\infty, T=0) \simeq 1.2 \pm 0.1 \mathrm{GeV}$ [13]. This result is quite universal; it is obtained by separating a heavy quark-antiquark pair, where the separation requires the formation of a light $q \bar{q}$ pair to assure color neutrality. It is obtained as well even if we separate a heavy quark-quark pair, where the formation of a light antiquark-antiquark pair is necessary [14]. Moreover, it is reached for any color channel (singlet or octet for $Q \bar{Q}$, antitriplet or sextet for $Q Q$ ). The large $r$ behavior of all cases coincides; any uncertainty in the numerical value of $F(\infty, T=0)$ is due to the necessity to extrapolate to $T=0$ and to uncertainties in the normalization. 
To create an isolated heavy-light quark system, we thus need a gluonic energy input $F_{g}$, with

$$
F(\infty, T=0)=2 F_{g} \simeq 1.2 \mathrm{GeV} .
$$

There are various ways to cross-check this result. If we consider a QCD medium in which light quarks are not so light, having a specified bare mass $m_{q}$, then the result should be

$$
F(\infty, T=0)=2 F_{g}+2 m_{q} .
$$

For a range of different values of $m_{q}$, one always obtains $F_{g} \simeq 0.6 \mathrm{GeV}$ [15. Lattice studies have also considered the quark separation of a nucleon, bringing all three quarks far apart [16]. The resulting free energy now becomes

$$
F_{3}(\infty, T=0)=3 F_{g},
$$

and the lattice results again lead to the same value of $F_{g}$ as found above.

Experimentally, a similar check can be performed, comparing the mass values of the open charm and open bottom mesons to those of the charm and bottom quarks. This yields

$$
\begin{aligned}
& M_{D}-m_{c}=F_{g}=0.60 \pm 0.10 \mathrm{GeV} \\
& M_{B}-m_{b}=F_{g}=0.53 \pm 0.15 \mathrm{GeV},
\end{aligned}
$$

using the relevant mass values as given by the PDG listing [25], and thus confirms that the resulting value does not depend on the quark source mass. The sizes of the heavylight mesons are expected to be of hadronic scale, so that a gluonic polarization system of spatial extent makes sense. The spatial extent of quarkonia, however, decrease inversely proportional to the heavy quark mass. Hence the $\Upsilon$ presents to the gluonic medium effectively a color singlet system of negligible extent, and it should therefore produce no polarization. In accord with this, one has

$$
M_{\Upsilon}-2 m_{b}=0.06 \pm 0.31 \mathrm{GeV},
$$

so that $\Upsilon$ the mass is essentially twice the bare bottom quark mass.

The same value persists for light-light quark systems, giving the correct vector meson masses (for both non-strange and strange ground states) as well as the correct different ground state baryon masses. In the case of hadrons containing strange quarks, the relevant number of strange quark masses $\left(m_{s} \simeq 100 \mathrm{MeV}\right)$ has to be added.

What is the meaning of $F_{g}$ ? One possible and rather widely accepted interpretation is that it is the "mass" or the energy content of the gluonic string connecting quark and antiquark. With

$$
F_{g} \simeq \sigma R_{h} \simeq 0.6-0.8 \mathrm{GeV}
$$

and using $\sqrt{\sigma}=0.4 \mathrm{GeV}$ and $R_{h}=0.8-1.0 \mathrm{fm}$, this does lead to the correct value of $F_{g}$, at least in the case of mesons; baryons are not so easily dealt with.

We want to consider here instead a scenario in which $F_{g}$ is the sum of the gluonic dressing masses of two constituent quarks. Then both mesons and baryons can be treated on equal footing, giving us

$$
M_{q}=\frac{F_{g}}{2}+m_{q} \simeq 0.3-0.4 \mathrm{GeV},
$$


where $m_{q}$ denotes the bare quark mass; the last term thus corresponds to the light quark limit. We emphasize that the constituent quarks retain their intrinsic quantum numbers; the gluon cloud thus is color-neutral and without any spin.

Such an interpretation is, as already mentioned, supported by the additive quark model 22. In a collision energy range of about $\sqrt{s} \simeq 5-20 \mathrm{GeV}$, in which hard processes do not yet play a significant role, the total cross sections for proton-proton and pion-proton collisions are given as

$$
\sigma_{p p}=3 \times 3 \sigma_{q q} \simeq 38 \mathrm{mb}, \quad \sigma_{\pi p}=2 \times 3 \sigma_{q q} \simeq 25 \mathrm{mb} .
$$

The predicted ratio $3 / 2$ between pion and proton projectiles is seen to be in accord with the data; moreover, $\sigma_{p p}=\pi R_{h}^{2}$ leads to $R_{h} \simeq 0.9 \mathrm{fm}$ for the hadronic radius. Using eq. (8), we obtain

$$
\sigma_{q q} \simeq 3.3 \mathrm{mb} \rightarrow R_{q} \simeq 0.33 \mathrm{fm}
$$

for the corresponding constituent quark sizes in the case of light bare quarks; we return to the more general case of $m_{q} \gg 0$ shortly. A similar constituent quark radius was also obtained through partonic arguments [24].

As mentioned, we consider the constituent quark to be made up of the bare quark and the gluonic polarization cloud surrounding it. This means that as we move a distance $r$ away from the pointlike quark, we find an effective quark mass $M_{q}^{\text {eff }}(r)$, depending on how much of the cloud we include at a given $r$. Screening in the non-abelian gluon medium limits the size of the cloud, so that beyond $r_{0} \simeq 0.3 \mathrm{fm}$, the cloud mass saturates, with the constituent quark mass $M_{q}$ as saturation limit. The resulting behavior [4] is illustrated in Fig. 2, with $R_{h} \simeq 1 \mathrm{fm}$ denoting the radius of color confinement.

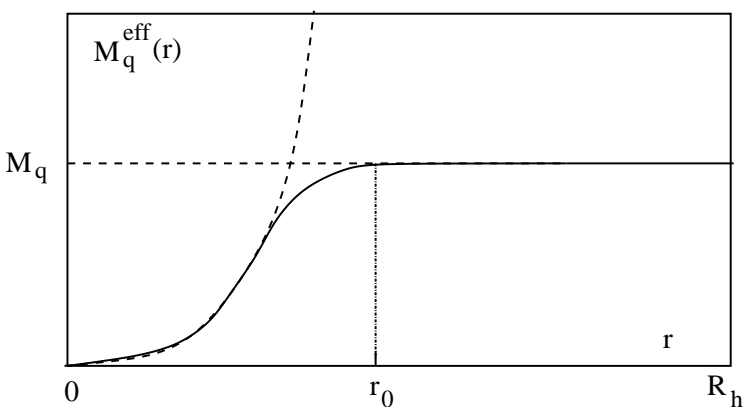

Figure 2: Effective quark mass $M_{q}^{\text {eff }}(r)$ as seen from a distance $r$ [4].

The conceptual scenario just discussed is supported by perturbative QCD estimates [26]. In the chiral limit $\left(m_{q} \rightarrow 0\right)$, the effective quark mass $M_{q}^{\text {eff }}(r)$ at scale $r$ is determined by the (non-perturbative) chiral condensate $\langle\bar{\psi} \psi\rangle$ and renormalization factors,

$$
M_{q}^{\mathrm{eff}}(r)=4 g^{2}(r) r^{2}\left[\frac{g^{2}(r)}{g^{2}\left(r_{0}\right)}\right]^{-d}\left\langle\bar{\psi} \psi\left(r_{0}\right)\right\rangle,
$$

where $r_{0}$ denotes a reference point for the determination of $\left\langle\bar{\psi} \psi\left(r_{0}\right)\right\rangle$; it is something like the meeting point of perturbative and non-perturbative regimes. For the case of massless 
quarks with $N_{c}=3, N_{f}=3$, the anomalous dimension is $d=4 / 9$, and

$$
g^{2}(r)=\frac{16 \pi^{2}}{9} \frac{1}{\ln \left[1 /\left(r^{2} \Lambda_{\mathrm{QCD}}^{2}\right)\right]}
$$

is the running coupling at scale $r$. The constituent quark mass $M_{q}$ is defined as the solution of eq. (10) at the scale $r=1 / 2 M_{q}$ [26]. This allows us to rewrite eq. (10) in the form

$$
M_{q}^{\mathrm{eff}}(r)=M_{q}\left[4 M_{q}^{2} r^{2}\right]\left[\frac{g^{2}\left(1 / 2 M_{q}\right)}{g^{2}(r)}\right]^{d-1}
$$

showing how the effective quark mass decreases at short distances $r<1 / 2 M_{q}$, starting from the constituent quark value. The value itself is determined by the non-perturbative chiral condensate at some reference point $r_{0}$. From eq. (12) we have

$$
\frac{M_{q}^{3}}{\left\langle\bar{\psi} \psi\left(r_{0}\right)\right\rangle}=\left(\frac{16 \pi^{2}}{9}\right)\left(\ln \left[1 /\left(r_{0}^{2} \Lambda_{Q C D}^{2}\right)\right]\right)^{-4 / 9}\left(\ln \left[4 M_{q}^{2} / \Lambda_{Q C D}^{2}\right]\right)^{-5 / 9} .
$$

As reference scale we use $r_{0}=1 / 2 M_{q}$, since that is where the perturbative evolution stops and the non-perturbative regime starts. We thus obtain

$$
\frac{M_{q}^{3}}{\left\langle\bar{\psi} \psi\left(r_{0}\right)\right\rangle}=\frac{16 \pi^{2}}{9} \frac{1}{\ln \left(4 M_{q}^{2} / \Lambda_{Q C D}^{2}\right)},
$$

showing that the constituent quark mass indeed provides a scale parameter for chiral symmetry breaking. To get an estimate for its value, we use $\Lambda_{Q C D}=0.2 \mathrm{GeV}$ and $\left\langle\bar{\psi} \psi\left(r_{0}\right)\right\rangle^{1 / 3}=0.2 \mathrm{GeV}$; this yields as solution of eq. (14) $M_{q}=375 \mathrm{MeV}$; the corresponding constituent quark radius becomes $R_{q}=r_{0}=0.26 \mathrm{fm}$. At the point $r_{0}=1 / 2 M_{q}$, eq. (11) gives for the strong coupling

$$
\alpha_{s}\left(r_{0}\right)=\frac{g^{2}\left(r_{0}\right)}{4 \pi} \simeq 0.5
$$

we have assumed a perturbative evolution in $r$ up to this point, which may be subject to some doubt. The value (15) is in approximate agreement with the value obtained in static quark lattice studies [27], which indicate, however, remaining non-perturbative contributions at this $r$. Nevertheless, it seems remarkable that the results agree rather well with the estimates obtained above from lattice calculations as well as from experiment.

The constituent quark radius is thus found to be $R_{q}=1 / 2 M_{q}$ in the chiral limit. More generally, we then expect

$$
R_{q} \simeq \frac{1}{2\left(M_{q}+m_{q}\right)}
$$

leading to a decrease in size with increasing bare mass. This is in accord with the decreasing cross sections for the interaction of strange or charm mesons [28].

The effective constituent quark mass is thus determined by the size and energy density of the gluon cloud, or equivalently, by the chiral condensate value in the non-perturbative region. How do these quantities change with temperature in a hadronic medium at vanishing baryon density? 
This can again be deduced from heavy quark correlation studies as function of the temperature of the medium. They show that the effective mass of the gluon cloud of an isolated static color charge (obtained by separating a static $Q \bar{Q}$ pair), starting from confinement values around $300 \mathrm{MeV}$, drops sharply at $T \simeq T_{c}$ [21]. This is accompanied by a corresponding drop of the screening radius. We thus expect the effective quark mass to show the temperature dependence illustrated in Fig. 3 .
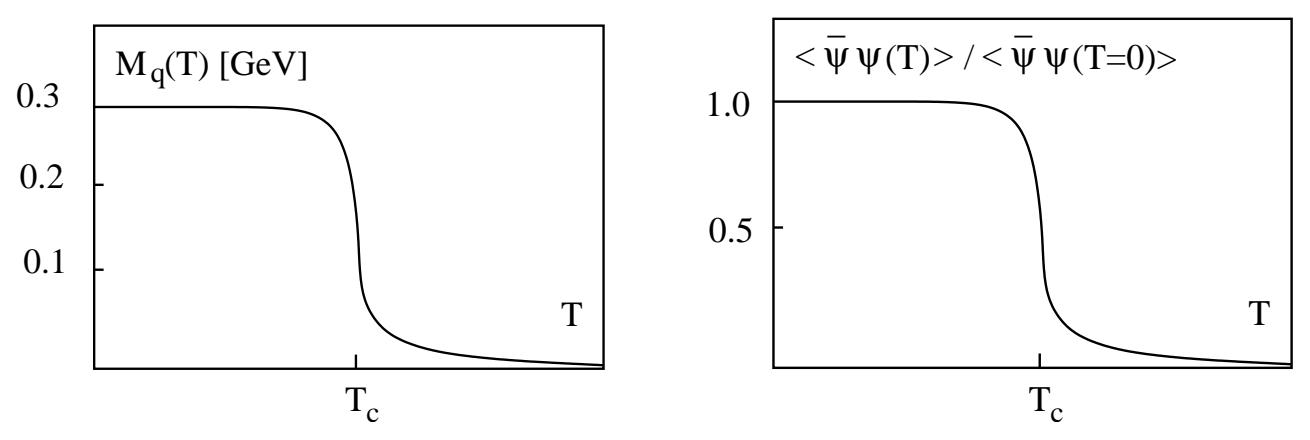

Figure 3: Constituent quark mass $M_{q}(T)$ (left) and chiral condensate $\langle\bar{\psi} \psi(T)\rangle$ (right) as function of temperature $T$.

Complementary to this, the temperature dependence of the chiral condensate is determined directly in finite temperature lattice QCD. Its behavior is also shown in Fig. 3: it is seen that at the deconfinement point, the chiral condensate vanishes as well [21]. This is in accord with the idea that at this point, the gluon cloud around the give quark has essentially evaporated.

These considerations show that there are two distinct ways to reach chiral symmetry restoration. On the one hand, even for an interquark distance $2 R_{h}$ well above $2 R_{q}$, a sufficiently hot medium will through gluon screening cause the effective quark mass to vanish, as shown in Fig. 3. On the other hand, when a cold medium becomes so dense that the average interquark distance is $2 R_{q}$ or less, the quarks form a connected cluster containing pointlike bare quarks.

We note in passing that the two scales, $R_{h}$ and $R_{q}$, have also been considered as the quark and gluon confinement scales, respectively. This implies that color-neutral hadrons have size $R_{h}$, whereas color-neutral glueballs have the much smaller intrinsic size $R_{q}$, and the spatial ground state glueball size is indeed in most calculations found to be about $R_{q} \simeq 0.3 \mathrm{fm}$.

We want to argue in the following section that at $\mu=0$, color deconfinement sets in at relatively large interquark separation, but in a hot gluonic environment. As a consequence, the gluon cloud giving rise to the effective quark mass has evaporated, causing deconfinement and chiral symmetry restoration to coincide. For the other extreme, for $T \simeq 0$, at the color deconfinement point the interquark distance is also still well above $R_{q}$. But now there is no hot gluonic medium to melt the gluon cloud, so that the cold deconfined medium will be a plasma of massive quarks. Only when its density is increased much more, to the percolation point of the massive constituent quarks, will the medium effectively consist of massless pointlike quarks. 


\section{Percolation and Phase Boundaries}

Hadrons are color-singlet quark-antiquark or three-quark states having a characteristic spatial extension of about one fermi. In a hadronic medium, the quark constituents are restricted to the corresponding volume by color confinement, making it impossible for a given quark to separate more than about a fermi from its partner(s) in a colorsinglet. When the density of a gas of hadrons is increased sufficiently, by raising either the temperature or the baryon density, a quark constituent in a given hadron will eventually find quarks and antiquarks from other hadrons as close by as its original partner(s). At this point, one can no longer define specific hadrons and hence the concept of hadronic matter becomes meaningless; the hadron gas has become a plasma of deconfined colored quarks and antiquarks. This is illustrated schematically in Fig. 4 for a discretized world. As mentioned, such a density limit to hadronic matter was first suggested by Pomeranchuk, well before the advent of the quark model of hadrons [19].
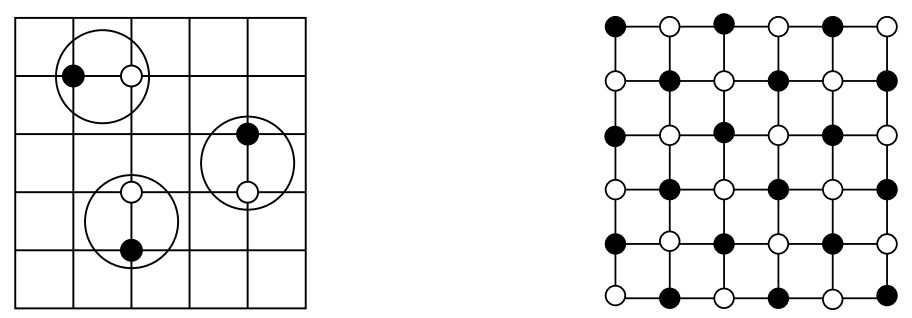

Figure 4: Schematic view of the transition from confined (left) to deconfined matter (right)

In the chiral limit, both mesons and baryons have an intrinsic spatial size of about $1 \mathrm{fm}$. Hence in both cases, the formation of percolating clusters provides a natural limit to the hadronic form of strongly interacting matter [29 32]. We note that for spin dynamics, the resulting percolation theory can be rigorously formulated [33,34]; for QCD, however, there were only some first approximative attempts [35, and a definitive theory is still lacking. In particular, both the definition of clusters (using bond weights) and the relevant thermal distribution law (with more than next neighbors) are not yet specified. Our argumentation thus has to remain on a qualitative level.

For mesons, increasing the density eventually leads to a medium in which the distance between a quark constituent from one hadron and an antiquark from another is equal to or less than the typical hadronic size, so that defining specific quark-antiquark pairs as hadrons ceases to make any sense. The percolating medium becomes a plasma of deconfined quarks, antiquarks and gluons. In the baryon-rich region, the increase of density of hard-core nucleons leads to "jamming", i.e., a restriction in the mobility of the nucleons [36, 37]. But here as well we reach eventually the formation of a percolating medium [38], in which the overall quark density is too high to define individual nucleons. We thus consider the limit of confinement in the $T-\mu$ diagram of strongly interacting matter to be universally defined by percolation of the relevant hadron species in the given region [32. It should be emphasized that such a percolation limit is a well-defined geometric form of critical behavior, specified by critical exponents and leading to universality classes, just as found for thermal critical behavior [20]. The essential difference is that ge- 
ometric singularities (formation of infinite clusters) do not necessarily imply non-analytic behavior for the partition function.

At $\mu=0$, the percolation density for permeable hadrons (mesons and low density baryons) of size $V=4 \pi R_{h}^{3} / 3$ is found to be 32 ]

$$
n_{M} \simeq \frac{1.2}{V_{h}} \simeq 0.6 \mathrm{fm}^{-3}
$$

with $R_{h} \simeq 0.8 \mathrm{fm}$ for the hadron radius. This is the density of the percolating cluster at the onset of percolation, or, equivalently, the density at which the medium no longer allows a spanning vacuum. The corresponding temperature can be obtained once the hadronic medium is specified. For $\mu \simeq 0$, this medium shows abundant resonance formation, and such interactions can be taken into account [39,40] by replacing the interacting medium of pions and nucleons by an ideal gas of all observed resonance states. For such an ideal gas one finds as deconfinement temperature 32]

$$
T_{\mathrm{dec}} \simeq 180 \mathrm{MeV}
$$

which agrees well with the value presently obtained in finite temperature lattice QCD.

The resulting medium at this point is, however, strongly non-perturbative. In fact, if we consider as above (see eq. (15)) the onset of perturbative behavior to be given by $\alpha_{s} \simeq 1 / 2$, the non-perturbative regime extends up to $T \simeq 4 T_{c}$. This is again in accord with lattice results, showing that above $3-4 T_{c}$ the interaction measure $(\epsilon-3 P) / T^{4}$ reaches perturbative behavior [41].

Assuming mesons to be the dominant constituents, the density (17) implies for the average separation between quarks and/or antiquarks at the deconfinement point

$$
d_{q}^{M} \simeq \frac{1}{n_{M}^{1 / 3}} \simeq 1.2 \mathrm{fm},
$$

as illustrated in Fig. 4 .

At the other extreme, for $T=0$, we have to consider the percolation of nucleons with a hard core [32,38]. Assuming a hard core radius $R_{\mathrm{hc}}=R_{h} / 2$, one obtains for the critical density

$$
n_{B} \simeq \frac{2}{V_{h}} \simeq 0.9 \mathrm{fm}^{-3}
$$

a value about $30 \%$ higher than that for permeable hadrons, as consequence of the baryon repulsion. This value can be used to obtain the percolation value of the baryochemical potential, using a van-der-Waals approach to account for the repulsion in the determination of the density as function of $\mu[32]$. As result, one obtains

$$
\mu_{\mathrm{dec}} \simeq 1.1 \mathrm{GeV}
$$

as deconfinement point for $\mu$. The separation between quarks at this point becomes

$$
d_{q}^{B} \simeq \frac{1}{n_{B}^{1 / 3}} \simeq 1.0 \mathrm{fm}
$$


slightly less than at $T=0$, due to the higher density. We note that since $\mu \geq M$, where $M$ denotes the nucleon mass, this leaves as function of $\mu$ a rather small window

$$
M \leq \mu \leq 1.2 M
$$

for the range of confined baryonic matter at $\mathrm{T}=0$. This window contains essentially all strongly interacting matter in the real world, from nuclei to neutron stars. The corresponding density range runs from $n_{0} \simeq 0.17 \mathrm{fm}^{-3}$ as standard nuclear matter density to the deconfinement value (20) of about $5 n_{0}$.

We thus confirm through percolation arguments that color deconfinement is expected to set in at hadron densities for which in general the quark constituents are separated by about $1 \mathrm{fm}$. At this density, any partitioning into hadrons becomes meaningless, and we have a medium of deconfined quarks of mass $M_{q} \simeq 0.4 \mathrm{GeV}$ and size $r_{0} \simeq 0.3 \mathrm{fm}$, separated by a distance $r \simeq 1 \mathrm{fm}>r_{0}$. Hence in the density range corresponding to $r_{0} \leq r \leq 1 \mathrm{fm}$, the quarks can retain their effective constituent mass, so that the deconfined medium now is a plasma of quarks of finite mass and spatial extent, with continued chiral symmetry breaking. A sufficient further increase in density will eventually lead to overlap and percolation of the constituent quarks. We assume that beyond this percolation point, chiral symmetry is effectively restored. Let us see what density the above obtained value of $R_{q}$ leads to.

At $T=0$, the density for a system of quarks of mass $M_{q}$ is given by

$$
n_{q}\left(\mu_{q}\right)=\frac{2}{\pi^{2}}\left(\mu_{q}^{2}-M_{q}^{2}\right)^{3 / 2},
$$

with $\mu_{q}=\mu / 3$ for the quark chemical potential. The percolation condition for quarks of radius $R_{q}$ (see eq. (17))

$$
n_{q}^{\mathrm{ch}}=\frac{1.2}{\left(4 \pi R_{q}^{3} / 3\right)} \simeq \frac{0.29}{R_{q}^{3}}
$$

then defines the onset of chiral symmetry restoration. With $R_{q} \simeq 0.3 \mathrm{fm}$, we obtain

$$
n_{B}^{\mathrm{ch}} \simeq 3.5 \mathrm{fm}^{-3} \simeq 3.9 n_{B}^{\mathrm{dec}}
$$

indicating that the baryon density threshold for chiral symmetry restoration is about four times higher than that for color deconfinement. The corresponding value for the baryochemical potential is found to be $\mu_{B}^{\text {ch }} \simeq 2.2 \mathrm{GeV}$, to be compared to $\mu_{B}^{\text {dec }} \simeq 1.1 \mathrm{GeV}$. Using the $\mu$ counterpart of the two-loop form (11), the strong coupling $\alpha_{s}$ has now dropped to the value $\alpha_{s}\left(\mu_{c}^{\mathrm{ch}}\right) \simeq 0.5$. The resulting phase structure is schematically illustrated in Fig. 5.

We close this section with some comments on the origin of the limiting curves. The hadronic phase ends both in $T$ and in $\mu$ by percolation, for permeable mesons and hardcore nucleons, respectively. The temperature limit coincides with the gluon evaporation limit, at which the constituent quark mass vanishes in a non-perturbative way. This presumably will also occur in the phase of massive quark constituents, so that the temperature limit of that phase is defined by gluon evaporation. The low temperature density 


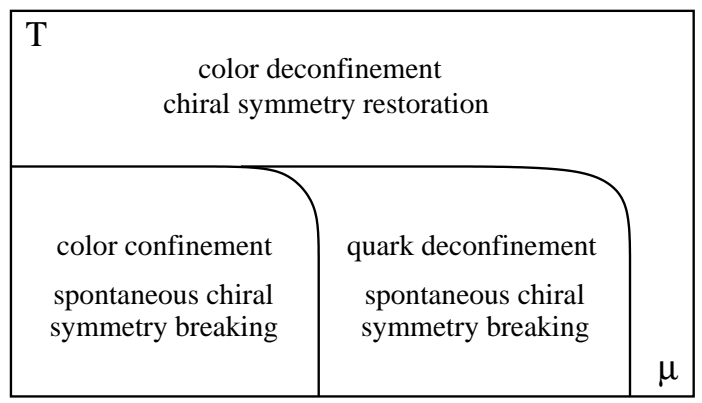

Figure 5: Phase structure of strongly interacting matter

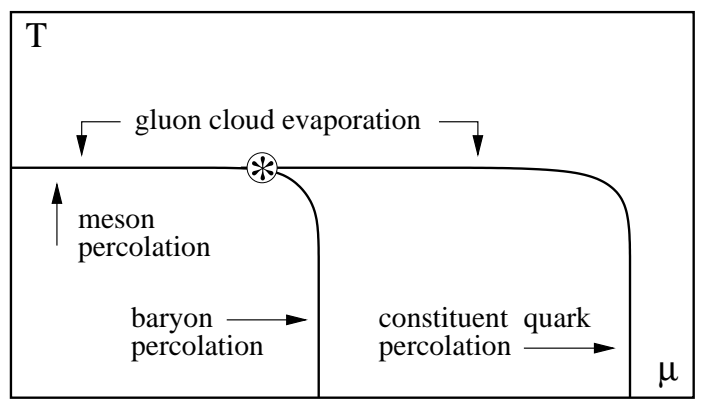

Figure 6: Limits of strongly interacting matter

limit there, however, is determined by constituent quark percolation. The behavior in the different regions is summarized in Fig. 6.

The nature of the limits for the hadronic phase, as given in a percolation picture, was elaborated in [32]. The mesonic percolation limit is in general purely geometric and not a thermal phase transition, although given specific dynamics, it can be [33, 34]. The form of the restoration of chiral symmetry there depends on $N_{f}$. The percolation of hard-core nucleons in the presence of an overall nucleon attraction leads to a Van der Waals situation and thus to a first order phase transition [32, ending in a second order point $T^{*}, \mu^{*}$. In view of the adjacent constituent quark phase this can become a triple point, as illustrated in Fig. 6] [17, 18].

\section{The Structure of a Plasma of Massive Quarks}

Our considerations thus suggest the existence of a plasma of massive deconfined quarks between the hadronic matter state and the quark-gluon plasma. In this state, quarks are deconfined; gluons, however, are "bound" into the constituent quark mass and thus remain in a sense confined. The quark dressing is made up of gluons which form a colorneutral cloud, so that the massive quarks retain their fundamental color state as well as their other intrinsic quantum numbers. The effective degrees of freedom in the resulting quark plasma thus are just those of massive quarks. Its lower limit in baryon density is defined by the onset of vacuum formation, forcing the quarks to bind into color neutral nucleons. The corresponding high density limit is given by the percolation point of the (spatially extended) quarks, beyond which there is a connected medium containing bare quarks and gluons. Finally, increasing the temperature at fixed $\mu$ leads here, just as in 
the hadronic phase, to an evaporation of the gluonic dressing of the quarks and thus to a restoration of chiral symmetry.

The constituent quarks in the deconfined medium will in general be interacting with each other. Of particular interest here is the presence of a $q q$ attraction, which at sufficiently low temperatures could lead to the formation of bound colored bosonic $q q$ states ("diquarks"). Baryons have in fact often been considered in terms of two quarks bound in a color antitriplet state, which then in turn binds with the remaining quark to form a color singlet. For $T \simeq 0$, extensive studies have addressed the formation of QCD Cooper pairs; their condensation would then lead to a color superconductor [3]. In contrast to electromagnetic superconductors, where only the global background field (phonons) of the medium can result in a binding of electrons into Cooper pairs, we have in QCD a local $q q$ color anti-triplet attraction, provided by gluon exchange. Hence diquarks could here exist as local "particle" bound states and not just as momentum state pairs of undefined spatial separation. The thermodynamics of a medium of such "localizable" diquarks was in fact considered some time ago [42 44] 1 Moreover, heavy quark studies [14] indicate that the interquark potential for a color anti-triplet $Q Q$ pair in a deconfined medium is attractive and essentially identical to that for a color singlet $Q \bar{Q}$ pair.

To reach baryon densities beyond the percolation limit, the nucleons evidently have to break up into constituents which are subject to less or no hard-core repulsion, and a break-up into constituent quarks and bosonic diquarks as their excited states fills that requirement. Since at low $T$ there is little or no thermal agitation, both could survive for some range of temperature and baryochemical potential. The resulting picture of the new medium thus parallels somewhat that of hadronic matter at $\mu \simeq 0$, where resonance interactions lead to a gas of basic hadrons (pions and nucleons) plus their resonance excitations. Here we have instead a gas of basic constituent quarks, together with the diquark excitations formed through their interaction 2 . The essential difference is that the basic "particles" now are massive colored fermions, which can exist only in the colored background field provided by a sufficiently dense strongly interacting medium.

Let us elaborate this structural similarity a little and look at the possible limits of the two different states of matter with broken chiral symmetry, hadron gas and constituent quark gas. In the case of hadronic matter at low $\mu$, we had obtained a limit through percolation, based on the geometric size of hadrons and their overlap [19]. Such a limit will presumably exist as well for constituent quarks and diquarks of comparable size. For constituent quarks, overlapping gluon clouds prevent an unambiguous definition, and for diquarks, the presence of equally close alternative binding partners has the same effect. We thus expect a continuation of the percolation line into the deconfined state at larger $\mu$, defining here as well the limit of chiral symmetry breaking.

An alternative approach to limiting hadronic matter [45, 46] is based on the resonance structure of hadronic interactions. It leads to an exponentially increasing resonance degeneracy, which in turn produces singular thermodynamic behavior. The basis for this

\footnotetext{
${ }^{1}$ It should be noted, however, that possible observable consequences (H-dibaryon, diquark contributions to hadronic structure functions from deep inelastic scattering) in the low baryon density region have not been found.

${ }^{2}$ We restrict ourselves here to diquarks. In principle, the formation of multi-quark clusters seems also conceivable.
} 
is the possibility of replacing an interacting hadron gas by an ideal gas of all possible resonances [39, 40], provided that the interaction is specified by resonance formation. The limiting temperature is in this case determined by the range of the interaction [47,48]. If the form of the interaction in a color antitriplet state were similar to that in a color singlet state, we would expect diquarks to exhibit a corresponding resonance pattern. The resulting thermodynamics would then lead to a limiting temperature for the quark/diquark resonance gas, just as it did for the hadronic resonance gas 3 . We note that in both cases, the geometric argumentation appears as the more general one, since even a gas containing only extended ground state hadrons leads through percolation to a limit [19].

We thus speculate that the chiral symmetry breaking limit for the constituent quark state is in nature very similar to that of the hadronic state, reaching this conclusion either in terms of the percolation of spatially extended colored or colorless constituents, respectively, or through a conjectured resonance interaction pattern for diquark binding as well as for the hadron spectrum.

\section{Quark Plasma vs. Quarkyonic Matter}

We have argued that nuclear matter, with color confinement and chiral symmetry breaking, is separated from the canonical plasma of massless quarks (plus some gluons and antiquarks at $T \neq 0$ ) by an intermediate phase of massive quarks as basic constituents. Recent arguments dealing with strongly interacting matter in the large $N_{c}$ limit have introduced "quarkyonic" matter as an intermediate phase. Let us try to understand how such a state could be related to the massive quark plasma. We must keep in mind, however, that as long as corresponding features of quarkyonic matter are still under discussion, any comparison will remain tentative.

Let us first consider the relevant color degrees of freedom, assuming QCD with $S U\left(N_{c}\right)$ as underlying theory. In a hadron gas, with color singlet constituents, we have only one color state, $d_{\mathrm{eff}}^{c}=1$. In the constituent quark gas at large $\mu$, there are massive colored quarks, as well as some massive colored antiquarks for $T>0$, but no free gluons; hence here we have $d_{\text {eff }}^{c} \sim N_{c}$. Finally, in the quark-gluon plasma, we have $d_{\text {eff }}^{c}=2 N_{c}+\left(N_{c}^{2}-1\right)$, with the first term counting quarks and antiquarks, the second gluons. For large $N_{c}$, the three states of matter thus behave as $1: N_{c}: N_{c}^{2}$, respectively, and in this aspect our picture results in a behavior similar to that found in the quarkyonic matter approach.

However, the limit of large $N_{c}$ results in several rather drastic modifications of the phase diagram. The baryon density in the hadronic regime is given by

$$
n_{B} \sim \exp \{(\mu-M) / T\}
$$

where $M$ again denotes the nucleon mass. Since both $M$ and $\mu$ are linear in $N_{c}$, the nuclear matter region is in the large $N_{c}$ limit contracted to $\mu=M$. The hadronic regime thus becomes purely mesonic. Similarly, the quark-gluon plasma, with

$$
T \ln Z\left(T, \mu_{q}, V\right)=\left(N_{c}^{2}-1\right)\left(\frac{\pi^{2} V T^{4}}{45}\right)+N_{f} N_{c}\left(\frac{V}{6}\right)\left[\left(\frac{7 \pi^{2} T^{4}}{30}\right)+\mu_{q}^{2} T^{2}+\left(\frac{\mu_{q}^{4}}{2 \pi^{2}}\right)\right],
$$

\footnotetext{
${ }^{3}$ The limiting temperature of the hadronic resonance gas is determined by the range of the interaction and persists even in the chiral limit of massless pions. In the original Hagedorn formulation [45, the estimate $T_{H} \sim m_{\pi}$ arose by assuming the pion mass to determine the interaction range.
} 
becomes for large $N_{c}$ gluon dominated for all finite $\mu$ and $T \geq T_{c}$. We thus obtain a phase diagram featuring mesonic matter for $T \leq T_{c}, \mu<M$, and a gluon plasma for $T \geq T_{c}$ and all $\mu$. The remaining section, with $T \leq T_{c}$ and $\mu \geq M$, is the regime of the proposed quarkyonic matter. Its effective color degrees of freedom are $d_{\text {eff }}^{c} \sim N_{c}$ and it has non-vanishing baryon density; it must thus consist of deconfined quarks and confined gluons, either as quark dressing or as glueballs. In view of these alternatives, the question of chiral symmetry restoration remains open. The resulting large $N_{c}$ phase diagram is illustrated schematically in Fig. 7.

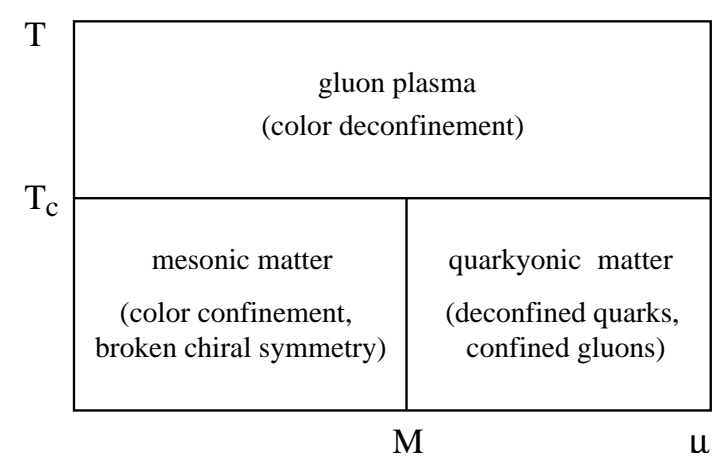

Figure 7: Phase structure of strongly interacting matter for large $N_{c}$

Our phase diagram thus has several features in common with that obtained for large $N_{c}$. An essential difference is that we assume the onset of chiral symmetry restoration in the quark plasma phase, at the percolation limit of the extensive massive quarks. With the quark density given by (see eq. (24))

$$
n_{q}=\frac{2 N_{c}}{3 \pi^{2}}\left(\mu_{q}^{2}-M_{q}^{2}\right)^{3 / 2},
$$

the percolation condition $n_{q}=1.2 / V_{q}$ contracts the $\mu$ range for $N_{c} \rightarrow \infty$ to the point $\mu_{q}=M_{q}$, or equivalently to $\mu=M$. In the limit of large $N_{c}$, our massive quark phase thus shares the fate of nuclear matter: everything is shrunk into one point, $\mu=M$.

\section{Conclusions}

Spontaneous chiral symmetry breaking gives the quarks in hadrons an effective "constituent" mass. We have argued that restoration can occur in two ways,

- by an evaporation of the gluonic quark dressing in a hot environment, or

- by quark percolation in a cold environment, leading to cluster fusion of the gluon clouds making up the effective quark mass.

We suggest that the coincidence of color deconfinement and chiral symmetry restoration at vanishing baryon density arises through the first of these two mechanisms. For low temperature and large baryochemical potential, an evaporation of the gluon dressing does not appear likely, and hence the effective quark mass will survive quark deconfinement. We thus obtain a three-phase structure of matter in QCD (apart from a possible color superconductor), consisting of 
- hadronic matter, with the hadronic size as intrinsic scale, confined quarks and gluons and broken chiral symmetry,

- a plasma of deconfined massive quarks, with their mass $\left(\sim\left\langle\bar{\psi} \psi\left(r_{0}\right)\right\rangle^{1 / 3}\right)$ as intrinsic scale, confined gluons, and broken chiral symmetry,

- a plasma of massless quarks and gluons, with no intrinsic scale, full color deconfinement and chiral symmetry restoration.

The phase of massive quarks is limited in temperature by evaporation, i.e., also by the first of the above alternatives, and in baryon density by the second, i.e., by quark percolation. In addition to basic massive quarks, the quark plasma can also contain diquarks as local quark-quark bound states in the colored background.

\section{Acknowledgements}

It is a pleasure to acknowledge helpful and stimulating discussions and/or correspondence with O. Kaczmarek, F. Karsch, L. McLerran and K. Redlich.

\section{References}

[1] See e.g. S. Reddy, Acta Phys. Polon. B33 (2002) 4101.

[2] See e.g., A. Kurkela, P. Romatschke and A. Vuorinen, arXiv:0912.1856.

[3] See e.g. M. Alford, K. Rajagopal, T. Schäfer and A. Schmitt, Rev. Mod. Phys. 80 (2008) 1455.

[4] E. V. Shuryak, Phys. Lett. 107B (1981) 103; Phys. Rep. 115 (1984) 151.

[5] R. Pisarski, Phys. Lett. 110B (1982) 155.

[6] G. Baym, in Quark Matter and Heavy Ion Collisions, H. Satz (Ed.), World Scientific, Singapore 1982

[7] J. Cleymans, K. Redlich, H. Satz and E. Suhonen, Z. Phys. C 33 (1986) 151.

[8] H. Schulz and G. Röpke, Z. Phys. C 35 (1987) 379.

[9] A. Drago, M. Fiolhais and U. Tambini, Nucl. Phys. A 588 (1995) 801.

[10] H. Toki and K. Suzuki, Progr. Theoret. Phys. 87, (1992) 1435.

[11] P. Guo and A. P. Szczepaniak, arXiv:0902.131 [hep-ph].

[12] T. Banks and A. Casher, Nucl. Phys. B 169 (1980) 103.

[13] O. Kaczmarek and F. Zantow, Phys. Rev. D 71 (2005) 114510.

[14] M. Döring, K. Hübner, O. Kaczmarek and F. Karsch, Phys. Rev. D 75 (2007) 05404. 
[15] F. Karsch, E. Laermann and A. Peikert, Nucl. Phys. B 605 (2001) 579.

[16] K. Hübner, F. Karsch, O. Kaczmarek and O. Vogt, Phys. Rev.

[17] L. McLerran and R. Pisarski, Nucl. Phys. A 796 (2007) 83.

[18] See A. Andronic et al., arXiv:0911.4806 [hep-ph], for a recent summary and further references

[19] I. Ya. Pomeranchuk, Doklady Akad. Nauk. SSSR 78 (1951) 889.

[20] See e.g.

D. Stauffer and A. Aharony, Introduction to Percolation Theory, Taylor and Francis, London 1994;

J. J. Binney, N. J. Dowrick, A. J. Fisher and M. E. J. Newman, The Theory of Critical Phenomena, Clarendon Press, Oxford 1992.

[21] For the most recent study and references to earlier literature, see

A. Bazavov et al., Phys. Rev. D 80 (2009) 014504.

[22] E. M. Levin and L. L. Frankfurt, JETP Letters 2 (1965) 65;

H. J. Lipkin and F. Scheck, Phys. Rev. Lett. 16 (1966) 71;

H. Satz, Phys. Rev. Lett. 19 (1967) 1453.

[23] For an early reference to such a picture, see e.g.

V. A. Novikov, M. A. Shifman, A. I. Vainshtein and V. I. Zakharov, Annals Phys. 105 (1977) 276.

[24] E. V. Shuryak and A. I. Vainshtein, Nucl. Phys. B 199 (1982) 451.

[25] C. Amsler et al. (Particle Data Group), Phys. Lett. B667 (2008) 1.

For $m_{c}$, we use the $\overline{M S}$ value at scale $2 \mathrm{GeV}$, which agrees with that found in potential model studies; for $m_{b}$, we use instead the $1 S$ value, which is closer to that found in potential model studies than the $\overline{M S}$ value at scale $2 \mathrm{GeV}$.

[26] H. D. Politzer, Nucl. Phys. B 117 (1976) 397.

[27] O. Kaczmarek, F. Karsch, P. Petreczky and F. Zantow, Phys. Rev. D 70 (2004) 074505.

[28] B. Povh and J. Hüfner, Phys. Rev. Lett. 58 (1987) 1612.

[29] G. Baym, Physica 96 A (1979) 131.

[30] T. Çelik, F. Karsch and H. Satz, Phys. Lett. 97 B (1980) 128.

[31] V. Magas and H. Satz, Europ. Phys. J. C 32 (2003) 115.

[32] P. Castorina, K. Redlich and H. Satz, Europ. Phys. J. C 59 (2009) 67.

[33] C. M. Fortuin, P. W. Kasteleyn J. Phys. Soc. Japan 26 (Suppl.), 11 (1969);

Physica 57, 536 (1972). 
[34] A. Coniglio and W. Klein, J. Phys. A 13 (1980) 2775.

[35] S. Fortunato and H. Satz, Phys. Lett. B 475 (2000) 311;

S. Fortunato, F. Karsch, P. Petreczky and H. Satz, Phys. Lett. 502 (2001)321.

[36] F. Karsch and H. Satz, Phys. Rev. D 21 (1980) 1168.

[37] See e.g. G. Biroli, Nature Physics 3 (2007) 222.

[38] K. W. Kratky, J. Stat. Phys. 52 (1988) 1413.

[39] E. Beth and G. E. Uhlenbeck, Physica 4 (1937) 915.

[40] R. Dashen, S.-K. Ma and H. J. Bernstein, Phys. Rev. 187 (1969) 345.

[41] V. Goloviznin and H. Satz, Z. Phys. C 57 (1993) 671;

G. Boyd, J. Engels, F. Karsch, E. Laermann, C. Legeland, M. Lütkemeier and B. Petersson, Nucl. Phys. B 469 (1996) 419.

[42] S. Ekelin, in Strong Interactions and Gauge Theories, J. Tran Thanh Van (Ed.), Editions Frontières, Gif-sur Yvette, 1986.

[43] J. F. Donohue and K. S. Sateesh, Phys. Rev. D 38 (1988) 360.

[44] M. Anselmino, S. Ekelin, D. B. Lichtenberg and E. Predazzi, Rev. Mod. Phys. 65 (1993) 1199.

[45] R. Hagedorn: Nuovo Cim. Suppl. 3, 147 (1965);

Nuovo Cim. 56A, 1027 (1968)

[46] K. Bardakci and S. Mandelstam: Phys. Rev. 1841640 (1969);

S. Fubini and G. Veneziano: Nuovo Cim. 64A 811 (1969).

[47] K. Huang and S. Weinberg, Phys. Rev. Lett. 25 (1970) 855.

[48] H. Satz, Fortsch. Physik 33 (1985) 259. 